\title{
Entropy Product Formula for Gravitational Instanton
}

\author{
Parthapratim Pradhan \\ Department of Physics, Hiralal Mazumdar Memorial College For Women, Dakshineswar, Kolkata 700035, India \\ Correspondence should be addressed to Parthapratim Pradhan; pppradhan77@gmail.com
}

Received 9 October 2016; Revised 13 March 2017; Accepted 27 March 2017; Published 23 April 2017

Academic Editor: Torsten Asselmeyer-Maluga

Copyright (C) 2017 Parthapratim Pradhan. This is an open access article distributed under the Creative Commons Attribution License, which permits unrestricted use, distribution, and reproduction in any medium, provided the original work is properly cited. The publication of this article was funded by SCOAP ${ }^{3}$.

We investigate the entropy product formula for various gravitational instantons. We speculate that due to the mass-independent features of the said instatons they are universal as well as quantized. For isolated Euclidean Schwarzschild black hole, these properties simply fail.

\section{Introduction}

There has been a strong interest in microscopic interpretation of black hole $(\mathrm{BH})$ entropy [1-8] in terms of $D$-branes due to the work by Strominger and Vafa [9]. In $d$-dimension Euclidean quantum gravity, this entropy is due to the $(d-2)$ dimensional fixed point sets of the imaginary time translation Killing vector. There are many fixed point sets which can also give rise to $\mathrm{BH}$ entropy.

Previously, Area (or Entropy) product formula was evaluated for different class of BHs [10-28]. In some cases, the product formula is not mass-independent (universal) and in some cases the product formula is indeed mass-independent, that is, universal. To the best of the author's knowledge, there has been no attempt to compute the entropy product formula for gravitational instanton.

Thus, in the present work, we wish to investigate the entropy product formula for various gravitational instantons. Instantons are nonsingular and having imaginary time. They arise in quantum field theory (QFT) for evaluating the functional integral, in which the functional integral is Wick rotated and expressed as an integral over Euclidean field configurations. They are the solutions of the Euclidean Einstein equations. They have the signature of the form $(++++)$. There are two types of instantons discovered so far. One is an asymptotically locally flat (ALF), which was first discovered by Hawking in 1977 [29] and the other is an asymptotically locally Euclidean (ALE) instanton, discovered by Gibbons and Hawking in 1979 [30]. The examples of ALE classes are Flat space, Eguchi-Hanson [31], and multi-instanton [30].
The ALF class of solutions are asymptotically flat (AF) in $3 \mathrm{D}$ sense, the fourth, imaginary time, direction being periodic. The surfaces of large radii should be thought of as an $\mathcal{S}^{1}$ bundle over $\delta^{2}$. The product bundle corresponds to the AF solutions which include the Euclidean Schwarzschild and Euclidean Kerr solutions [32, 33]. The twisted bundles correspond to the multi-Taub-NUT solution [29], and the TaubBolt solution was first discovered by Page [34].

In our previous work $[18,27,28]$, we investigated the properties of inner and outer horizon thermodynamics of Taub-NUT (Newman-Unti-Tamburino) BH, Kerr-Taub-NUT $\mathrm{BH}$, and Kerr-Newman-Taub-NUT BH in four-dimensional Lorentzian geometry. The failure of first law of BH thermodynamics and Smarr-Gibbs-Duhem relation for Taub-NUT and Kerr-Taub-NUT BH in the Lorentzian regime gives the motivation behind this work. What happens when one can go from Lorentzian geometry to Riemannian geometry? This is the prime aim in this work. By studying the properties of these instantons what should be the effects on the $\mathrm{BH}$ entropy product formula due to the nontrivial NUT parameter?

In general relativity, the nontrivial value for the $\mathrm{BH}$ entropy is due to the presence of the fixed point set of the periodic imaginary time Killing vector. The fixed point set we considered here actually is the $\mathrm{BH}$ horizons $\left(\mathscr{H}^{ \pm}\right)$. Here $\mathscr{H}^{+}$ is called event horizon and $\mathscr{H}^{-}$is called the Cauchy horizon. In four-dimensional space-time, such fixed point sets are of two types, isolated points or zero-dimensional which we call NUTs and two surfaces or two-dimensional which we call Bolts. Thus one can think of Bolts as being the analogue of 
electric type mass-monopoles and the NUTs as being gravitational dyons endowed with a real electric type massmonopole and an imaginary magnetic type mass-monopole. The presence of magnetic type mass introduces a Dirac string like singularity in the space-time, the so called Misner string, which was first pointed out by Misner in his paper for the Lorentzian Taub-NUT space-time [35]. A Misner string is a coordinate singularity which can be considered as a manifestation of a "nontrivial topological twisting" [36] of the manifold $\left(M, g_{a b}\right)$. This twist is parametrized by a topological term, the NUT charge.

In our previous investigation [18], we have taken the metric in a Lorentzian space-time in $3+1$ split form as

$$
d s^{2}=-\mathscr{F}\left(d t+w_{i} d x^{i}\right)^{2}+\frac{\gamma_{i j}}{\mathscr{F}} d x^{i} d x^{j}
$$

In this work, we are interested in studying the metric in a Riemannian space-time which can be written in $3+1$ split form:

$$
d s^{2}=\mathscr{F}\left(d \tau+w_{i} d x^{i}\right)^{2}+\frac{\gamma_{i j}}{\mathscr{F}} d x^{i} d x^{j} .
$$

Here all quantities are independent of $t$ or $\tau$. The Wick rotation that transforms from one case to the other is by the transformation $t \mapsto i \tau$ and $w_{i} \mapsto i \omega_{i}$. F can be thought of as an electric type potential, and $\omega_{i}$ or $w_{i}$ as a magnetic type vector potential. The associated magnetic field is $H_{i j}=\partial_{i} \omega_{j}-\partial_{j} \omega_{i}$ and it is gauge invariant.

This should be used to define a magnetic monopole moment called the NUT charge $n$ [30]. If $n \neq 0$, the fibration should not be trivial. In the Lorentzian geometry, these fixed point sets are the two-dimensional Boyer bifurcation sets of event horizon $[5,37]$. On the other hand, in Riemannian geometry, these fixed point sets are of two types: zerodimensional point or NUTs and two-dimensional surfaces or Bolts [30]. A NUT possesses a pair of surface gravities $\kappa_{1}$ and $\kappa_{2} . p$ and $q$ are a pair of coprime integers such that $\kappa_{1} / \kappa_{2}=$ $p / q$. If $\kappa_{1} / \kappa_{2}$ is irrational, $p=q=1$. A NUT of type $(p, q)$ has a NUT charge of $n=\beta / 8 \pi p q$, where $\beta$ is the period of the imaginary time coordinate. Moreover, $n=Y \beta / 8 \pi$ for a Bolt of self-intersection number $Y$. It should be noted that, in the Riemannian case, the number of NUTs and Bolts is related to the Euler number $\chi$ and the Hirzebruch signature $\tau$ of the manifold $M$ by

$$
\chi=\sum_{\text {Bolts }} \chi_{i}+\sum_{\text {NUTs }} 1
$$

where $\chi_{i}$ is the Euler number for the $i$ th Bolt, and

$$
\tau=\sum_{\text {Bolts }} Y_{i} \csc ^{2} \theta-\sum_{\text {NUTs }} \cot p_{i} \theta \cot q_{i} \theta+\eta(0, \theta) .
$$

Equation (4) is valid for arbitrary $\theta . Y_{i}$ is the self-intersection number of the $i$ th Bolt, the $i$ th NUT is of type $\left(p_{i}, q_{i}\right)$, and $\eta(0, \theta)$ is a correction term which depends solely on the boundary. It should be mentioned that for multi-Taub-NUT and multi-instanton solutions there are $k$ NUTs of type $(1,1)$ and $\tau=k-1$; therefore

$$
\tau=\sum_{\text {Bolts }} Y_{i} \csc ^{2} \theta-\sum_{\text {NUTs }} \cot p_{i} \theta \cot q_{i} \theta+k \csc ^{2} \theta-1,
$$

where the AF boundary conditions should be $\eta(0, \theta)=0$ [33].

The structure of the paper is as follows. In Section 2, we have considered the Euclidean Schwarzschild BH. In Section 3, we have investigated the properties of self-dual TaubNUT instantons. In Section 4, we have studied the massindependent properties of Taub-Bolt instantons. In Section 5, we have described the properties of Eguchi-Hanson instantons. In Section 6, we have examined the properties of TaubNUT-AdS space-time. In Section 7, we have studied the entropy product formula for Taub-Bolt-AdS space-time and finally in Section 8, we have examined the product rules for Dyonic Taub-NUT-AdS and Taub-Bolt-AdS space-time.

\section{Euclidean Schwarzschild Metric}

To give a warm up, let us first consider the Schwarzschild $\mathrm{BH}$ (where we have used units in $G=c=1$ ) in Euclidean form as

$$
\begin{aligned}
d s^{2}= & \left(1-\frac{2 M}{r}\right) d \tau^{2}+\frac{d r^{2}}{(1-2 M / r)} \\
& +r^{2}\left(d \theta^{2}+\sin ^{2} \theta d \phi^{2}\right) .
\end{aligned}
$$

The apparent singularity at the event horizon $r_{+}=2 M$ can be removed by identifying $\tau$ with a period $\beta=8 \pi M[29,33]$. The radial coordinate has the range $2 M \leq r \leq \infty$. Then the topology of the manifold is $R^{2} \times \delta^{2}$. The isometry group is $O(2) \otimes O(3)$, where $O(2)$ corresponds to translations in the periodically identified imaginary time $\tau$ and $O(3)$ corresponds to rotations of the $\theta$ and $\phi$ coordinates.

The Killing vector $\partial_{\tau}$ has unit magnitude at large radius and has a Bolt on the horizon $r_{+}=2 M$ which is a 2 -sphere $\mathcal{S}^{2}$ of area

$$
A_{+}=16 \pi M^{2} \text {. }
$$

The surface gravity is given by

$$
\kappa_{+}=\frac{2 \pi}{\beta}=\frac{1}{4 M},
$$

and the $\mathrm{BH}$ temperature is

$$
T_{+}=\frac{\kappa_{+}}{2 \pi}=\frac{1}{8 \pi M} .
$$

Thus for an isolated Euclidean Schwarzschild $\mathrm{BH}$ the area product becomes

$$
A_{+}=16 \pi M^{2} \text {, }
$$

which tells us that the product is dependent on mass parameter and thus it is not universal. Also it is not quantized.

The Euclidean action derived in [29] is

$$
I=-\ln Z=4 \pi M^{2} .
$$


From that one can derive the entropy as in [29]

$$
S_{+}=-\left(\beta \frac{\partial}{\partial \beta}-1\right) \ln Z=4 \pi M^{2} .
$$

Thus the entropy product for isolated Euclidean Schwarzschild $\mathrm{BH}$ should be

$$
S_{+}=4 \pi M^{2} .
$$

Indeed, it is not universal and it is not quantized.

\section{Self-Dual Taub-NUT Instantons}

In this section we shall calculate the entropy product and area product of four-dimensional Taub-NUT space-time. It is of ALF. ALF metrics have a NUT charge, or magnetic type mass, $n$, as well as the ordinary electric type mass, $M$. The NUT charge is $\beta c_{1} / 8 \pi$, where $c_{1}$ is the first Chern number of the $U(1)$ bundle over the sphere at infinity, in the orbit space $\Xi$. If $c_{1}=0$, then the boundary at infinity is $\mathcal{S}^{1} \times \mathcal{S}^{2}$ and the space-time is AF. The $\mathrm{BH}$ metrics are saddle points in the path integral for the partition function. Thus, if $c_{1} \neq 0$, the boundary at infinity is a squashed $\mathcal{S}^{3}$, and the metric should not be analytically continued to a Lorentzian metric. The squashed $\mathcal{S}^{3}$ is the three-dimensional space on which the boundary conformal field theory (CFT) will be compactified, with $\beta$ identified with the inverse temperature; that is, $T=1 / \beta$.

Hawking [29] first given example of gravitational instanton was the self-dual Taub-NUT metric described by

$$
\begin{aligned}
d s^{2}= & \mathscr{F}(r)(d \tau+2 n \cos \theta d \phi)^{2}+\frac{d r^{2}}{\mathscr{F}(r)} \\
& +\left(r^{2}-n^{2}\right)\left(d \theta^{2}+\sin ^{2} \theta d \phi^{2}\right), \\
& \mathscr{F}(r)=\frac{r-n}{r+n} .
\end{aligned}
$$

It is ALF with a central NUT. The self-dual Taub-NUT instanton has $M=n$ and the anti-self-dual instanton has $M=-n$. The value $r=n$ is now a zero in $\mathscr{F}(r)$. The $(\theta, \phi)$ two-sphere has a zero area at $r=n$, so the zero in $\mathscr{F}(r)$ is a zerodimensional fixed point of $\partial_{\phi}$, a NUT.

In order to make the solution regular, we take the region $r \geq n$ and let the period of $\tau$ be $8 \pi n$. The metric has a NUT at $r=n$, with a Misner string running along the $z$-axis from the NUT out to infinity; that is, $n \leq r \leq \infty$.

We know from the idea of path integral formulation of quantum gravity that the Euclidean action is derived in [29]

$$
I=-\ln Z=4 \pi n^{2},
$$

where $Z$ is the partition function of an ensemble

$$
Z=\int[D g][D \phi] e^{-I(g, \phi)},
$$

with the path integral taken over all metrics $g$ and matter field $\phi$ that are appropriately identified with the period $\beta$ of $\tau$. Therefore the entropy should be derived as

$$
S=-\left(\beta \frac{\partial}{\partial \beta}-1\right) \ln Z=4 \pi n^{2} .
$$

It is indeed mass-independent and thus it is universal.
The surface gravity is calculated to be

$$
\kappa=\frac{2 \pi}{\beta}=\frac{1}{4 n} \text {. }
$$

Thus the $\mathrm{BH}$ temperature should be read off

$$
T=\frac{\kappa}{2 \pi}=\frac{1}{8 \pi n} .
$$

Now we see what happens in the above results for other instantons that are Taub-Bolt.

\section{Taub-Bolt Instantons}

The Taub-Bolt instanton is described by the metric [34]

$$
\begin{aligned}
d s^{2}= & \mathscr{G}(r)(d \tau+2 n \cos \theta d \phi)^{2}+\frac{d r^{2}}{\mathscr{G}(r)} \\
& +\left(r^{2}-n^{2}\right)\left(d \theta^{2}+\sin ^{2} \theta d \phi^{2}\right), \\
\mathscr{G}(r)= & \frac{(r-2 n)(r-n / 2)}{\left(r^{2}-n^{2}\right)}=\frac{\left(r-r_{+}\right)\left(r-r_{-}\right)}{(r+n)(r-n)} .
\end{aligned}
$$

It is a non-self-dual, noncompact solution of the vacuum Euclidean Einstein equations. In order to make the solution regular we are restricted to the region $r=r_{+} \geq 2 n$, and the Euclidean time has period $\beta=8 \pi n$. Asymptotically, the Taub-Bolt instanton behaves in a similar manner to the TaubNUT, so it is ALF. Since we are setting the fixed point at $r=r_{+}=2 n$, therefore the area of the $\mathcal{S}^{2}$ does not vanish there and the fixed point set is 2-dimensional; thus it is a Bolt of area

$$
A_{+}=12 \pi n^{2} .
$$

Thus the area product for Taub-Bolt instanton will be

$$
A_{+}=12 \pi n^{2} \text {. }
$$

Thus the area product is independent of mass and also quantized. Similarly, the action was calculated in [38]

$$
I=-\ln Z=\pi n^{2} .
$$

Thus the entropy was derived by the universal formula

$$
S_{+}=-\left(\beta \frac{\partial}{\partial \beta}-1\right) \ln Z=\pi n^{2} .
$$

It indicates that the entropy product should be universal and quantized.

\section{Eguchi-Hanson Instantons}

A noncompact instanton which is a limiting case of the TaubNUT solution is the Eguchi-Hanson metric [31],

$$
\begin{aligned}
d s^{2}= & \left(1-\frac{n^{4}}{r^{4}}\right)\left(\frac{r}{8 n}\right)^{2}(d \tau+4 n \cos \theta d \phi)^{2} \\
& +\frac{d r^{2}}{\left(1-n^{4} / r^{4}\right)}+\frac{r^{2}}{4}\left(d \theta^{2}+\sin ^{2} \theta d \phi^{2}\right) .
\end{aligned}
$$


The instanton is regular if we consider the region $r \geq n$, and let $\tau$ has period $8 \pi n$. The metric is ALE type. There is a Bolt of area at $r=n$ given by

$$
A=\pi n^{2},
$$

which gives rise to a Misner string along the $z$-axis. Thus the product is universal and should be quantized. The Euclidean action is derived in [38]

$$
I=0 \text {. }
$$

Thus entropy corresponds to

$$
S=\left(\beta \frac{\partial}{\partial \beta}-1\right) I=0 .
$$

We now turn our attention to the Taub-NUT and TaubBolt geometries in four-dimensional locally AdS space-time. The spacetimes have a global nontrivial topology due to the fact that one of the Killing vectors has a zero-dimensional fixed point set called NUT or a two-dimensional fixed point set called Bolt. Moreover, these four-dimensional spacetimes have Euclidean sections which can not be exactly matched to AdS space-time at infinity.

\section{Taub-NUT-AdS Space-Time}

In this section we shall consider the space-time which is only locally asymptotically AdS and with nontrivial topology. The metric on the Euclidean section of this family of solutions could be written as $[39,40]$

$$
\begin{aligned}
d s^{2}= & \mathscr{H}(r)(d \tau+2 n \cos \theta d \phi)^{2}+\frac{d r^{2}}{\mathscr{H}(r)} \\
& +\left(r^{2}-n^{2}\right)\left(d \theta^{2}+\sin ^{2} \theta d \phi^{2}\right),
\end{aligned}
$$

where

$$
\mathscr{H}(r)=\frac{\left(r^{2}+n^{2}\right)-2 M r+\ell^{-2}\left(r^{4}-6 n^{2} r^{2}-3 n^{4}\right)}{r^{2}-n^{2}}
$$

and $\ell^{2}=-3 / \Lambda$, with $\Lambda<0$ being the cosmological constant. Here $M$ is a (generalized) mass parameter and $r$ is a radial coordinate. Also, $\tau$, the analytically continued time, that is, Euclidean time, parametrizes a circle $\mathcal{S}^{1}$, which is fibered over the two-sphere $\delta^{2}$, with coordinates $\theta, \phi$. The nontrivial fibration is a consequence of a nonvanishing NUT parameter $n$.

There are some restrictions [41] for existence of a regular NUT parameter. Firstly, in order to ensure that the fixed point set is zero-dimensional, it is necessary that the Killing vector $\partial_{\tau}$ has a fixed point which occurs precisely when the area of the two-sphere is zero in size. Secondly, in order for the DiracMisner string [35] to be unobservable, it is necessary that the period of $\tau$ be $\beta=8 \pi n$. To avoid the conical singularity, we must check $\mathscr{H}^{\prime}\left(r_{+}=n\right)=1 / 2 n$. Thirdly, the mass parameter $M$ must be $M=n-4 n^{3} / \ell^{2}$. After simplifying the metric coefficients, we obtain

$$
\mathscr{H}(r)=\left(\frac{r-n}{r+n}\right)\left[1+\frac{(r-n)(r+3 n)}{\ell^{2}}\right]
$$

and the range of the radial coordinate becomes $n \leq r \leq \infty$. For our requirement, the Euclidean action for this space-time was calculated in $[42,43]$

$$
I=-\ln Z=4 \pi n^{2}\left(1-\frac{2 n^{2}}{\ell^{2}}\right),
$$

and the entropy will be

$$
S_{+}=\left(\beta \frac{\partial}{\partial \beta}-1\right) I=4 \pi n^{2}\left(1-\frac{6 n^{2}}{\ell^{2}}\right) .
$$

Thus the entropy product should be

$$
S_{+}=4 \pi n^{2}\left(1-\frac{6 n^{2}}{\ell^{2}}\right) .
$$

It is independent of mass parameter and does depend on NUT parameter and cosmological constant. Thus the entropy product is universal for Taub-NUT-AdS space-time. The Hawking temperature $T_{+}=1 / 8 \pi n$ is the same as Taub-NUT $\mathrm{BH}$. The first law of thermodynamics is also satisfied as $d M=$ $T_{+} d S$.

If we consider the extended phase space following our previous work [24] and in this framework, the cosmological constant $(\Lambda)$ should be treated as thermodynamical pressure, that is, $P=-\Lambda / 8 \pi=3 / 8 \pi \ell^{2}$, and its conjugate variable should be treated as thermodynamic volume, that is, $V_{+}=(4 / 3) \pi r_{+}^{3}$, where $r_{+}$is the horizon radius. Then one should interpret the ADM mass $M$ parameter not to be the energy; rather it should be interpreted as enthalpy $H=M=U+P V_{+}$of the gravitational thermodynamical system. Therefore the thermodynamic volume has been calculated in [44] for TaubNUT-AdS space-time:

$$
V_{+}=\left(\frac{\partial H}{\partial P}\right)_{S}=-\frac{8}{3} \pi n^{3} .
$$

One aspect is that this is a peculiar result in a sense that the thermodynamic volume is negative and the other aspect is that the thermodynamic volume is universal because it is independent of the ADM mass parameter. It should be noted that the first law is fulfilled in this case and it yields

$$
d H=T_{+} d \mathcal{S}+V_{+} d P .
$$

Analogously, the Smarr-Gibbs-Duhem relation should be

$$
H=2 T_{+} \mathcal{S}-2 P V_{+},
$$

and in another interesting result we first claimed that the internal energy for Taub-NUT-AdS BH is universal. It is given by

$$
U=n\left(1-8 \pi P n^{2}\right)
$$

\section{Taub-Bolt-AdS Space-Time}

For Taub-Bolt-AdS, the metric has the same form as in (29) but the fixed point set here is two-dimensional or Bolt 
and with additional restrictions the metric coefficients $\mathscr{H}(r)$ vanish at $r=r_{b}>n$. In order to have a regular Bolt at $r=r_{b}$, the following conditions must be satisfied: (i) $\mathscr{H}\left(r_{b}\right)=0$, (ii) $\mathscr{H}^{\prime}\left(r_{b}\right)=1 / 2 n$, and the numerator of $\mathscr{H}(r)$ at $r=r_{b}$ being a single one. From condition (i), we get the mass parameter at $r=r_{b}$ :

$$
M=M_{b}=\frac{r_{b}^{2}+n^{2}}{2 r_{b}}+\frac{1}{2 \ell^{2}}\left(r_{b}^{3}-6 n^{2} r_{b}-3 \frac{n^{4}}{r_{b}}\right) .
$$

Then we find [41]

$$
\mathscr{H}^{\prime}\left(r_{b}\right)=\frac{3}{\ell^{2}}\left(\frac{r_{b}^{2}-n^{2}+\ell^{2} / 3}{r_{b}}\right) .
$$

To satisfy condition (ii) we must have the quadratic equation for $r_{b}$ :

$$
6 n r_{b}^{2}-\ell^{2} r_{b}-6 n^{3}+2 n \ell^{2}=0
$$

which gives the solution for $r_{b}$ in two branches:

$$
r_{b \pm}=\frac{\ell^{2}}{12 n}\left(1 \pm \sqrt{1-48 \frac{n^{2}}{\ell^{2}}+144 \frac{n^{4}}{\ell^{4}}}\right) .
$$

The discriminant of the above equation must be negative for $r_{b}$ to be real and for $r_{b}>n$ we obtain the following inequality for $n$ :

$$
n \leq n_{\max }=\sqrt{\frac{1}{6}-\frac{\sqrt{3}}{12}} \ell .
$$

The Euclidean action was computed in [43]

$$
I=\frac{4 \pi n}{\ell^{2}}\left(M_{b} \ell^{2}+3 n^{2} r_{b}-r_{b}^{3}\right)
$$

Now the entropy via the universal entropy formula can be easily derived:

$$
S_{+}=\left(\beta \frac{\partial}{\partial \beta}-1\right) I=4 \pi n\left(M_{b}-\frac{3 n^{2} r_{b}}{\ell^{2}}+\frac{r_{b}^{3}}{\ell^{2}}\right) .
$$

Now substituting the values of $\mathrm{M}_{b}$, we find the value of entropy

$$
S_{+}=4 \pi n\left[\frac{r_{b}^{2}+n^{2}}{2 r_{b}}+\frac{1}{2 \ell^{2}}\left(3 r_{b}^{3}-12 n^{2} r_{b}-3 \frac{n^{4}}{r_{b}}\right)\right] \text {. }
$$

Again, putting the values of $r_{b}$, we see that the entropy is universal as well as quantized.

\section{Dyonic Taub-NUT-AdS and \\ Taub-Bolt-AdS Space-Time}

The general form of the metric for dyonic Taub-NUT-AdS space-time [45-47] is given by

$$
\begin{aligned}
d s^{2}= & \mathcal{N}(r)(d \tau+2 n \cos \theta d \phi)^{2}+\frac{d r^{2}}{\mathcal{N}(r)} \\
& +\left(r^{2}-n^{2}\right)\left(d \theta^{2}+\sin ^{2} \theta d \phi^{2}\right),
\end{aligned}
$$

where

$$
\begin{aligned}
& \mathcal{N}(r) \\
& =\frac{\left(r^{2}+n^{2}+4 n^{2} v^{2}-q^{2}\right)-2 M r+\ell^{-2}\left(r^{4}-6 n^{2} r^{2}-3 n^{4}\right)}{r^{2}-n^{2}} .
\end{aligned}
$$

The gauge field reads off

$$
\begin{aligned}
A & \equiv A_{\mu} d x^{\mu} \\
& =\left(\frac{q r}{r^{2}-n^{2}}+\nu \frac{r^{2}+n^{2}}{r^{2}-n^{2}}\right)(d \tau-2 n \cos \theta d \phi) .
\end{aligned}
$$

The conditions of smoothness of the Euclidean section implies that the parameter $q$ is related to the parameter $v$ which gives a deformation from the uncharged system. When these parameters go to zero value, we obtain simply TaubNUT-AdS space-time.

In order to have a regular position of NUT or Bolt at $r=$ $r_{ \pm}$, we set $\mathcal{N}(r)=0$ and also the gauge field $A$ must be regular at that point. Thus we obtain the mass parameter as

$$
\begin{aligned}
M= & \frac{r_{ \pm}^{2}+n^{2}+4 n^{2} v^{2}-v^{2}}{2 r_{ \pm}} \\
& +\frac{1}{2 \ell^{2}}\left(r_{ \pm}^{3}-6 n^{2} r_{ \pm}-3 \frac{n^{4}}{r_{ \pm}}\right), \\
q= & -\frac{r_{ \pm}^{2}+n^{2}}{r_{ \pm}} \nu .
\end{aligned}
$$

The electric charge and potential at infinity correspond to

$$
\begin{aligned}
Q & =q, \\
\phi_{ \pm} & =-q \frac{r_{ \pm}}{r_{ \pm}^{2}+n^{2}}=v .
\end{aligned}
$$

Now the Euclidean action for the above space-time was calculated in [48] (in units where $G=c=1$ )

$$
\begin{aligned}
I_{ \pm} & =-2 \pi \\
& \cdot \frac{\left[r_{ \pm}^{4}-\ell^{2} r_{ \pm}^{2}+n^{2}\left(3 n^{2}-\ell^{2}\right)\right] r_{ \pm}^{2}-\left(r_{ \pm}^{4}+4 n^{2} r_{ \pm}^{2}-n^{4}\right) \ell^{2} v^{2}}{\left(3 r_{ \pm}^{2}-3 n^{2}+\ell^{2}\right) r_{ \pm}^{2}+\left(r_{ \pm}^{2}-n^{2}\right) \ell^{2} v^{2}} .
\end{aligned}
$$

The entropy was calculated as

$$
S_{ \pm}=2 \pi \frac{\left[3 r_{ \pm}^{4}+\left(\ell^{2}-12 n^{2}\right) r_{ \pm}^{2}+n^{2}\left(\ell^{2}-3 n^{2}\right)\right] r_{ \pm}^{2}+\left(r_{ \pm}^{4}+4 n^{2} r_{ \pm}^{2}-n^{4}\right) \ell^{2} v^{2}}{\left(3 r_{ \pm}^{2}-3 n^{2}+\ell^{2}\right) r_{ \pm}^{2}+\left(r_{ \pm}^{2}-n^{2}\right) \ell^{2} v^{2}}
$$


When we set $r_{ \pm}=r_{n}=n$, we get a dyonic NUT spacetime. For this spacetime the above calculations are reduced to

$$
\begin{aligned}
M & =n-\frac{4 n^{3}}{\ell^{2}}, \\
Q & =-2 n \nu, \\
\phi_{ \pm} & =\nu, \\
I_{ \pm} & =4 \pi n^{2}\left(1-2 \frac{n^{2}}{\ell^{2}}+2 \nu^{2}\right) .
\end{aligned}
$$

Finally, the entropy is given by

$$
S_{ \pm}=4 \pi n^{2}\left(1-6 \frac{n^{2}}{\ell^{2}}+2 \nu^{2}\right)
$$

Thus the entropy product formula for dyonic Taub-NUT is

$$
S_{+} S_{-}=S_{+}^{2}=S_{-}^{2}=\left(4 \pi n^{2}\right)^{2}\left(1-6 \frac{n^{2}}{\ell^{2}}+2 \nu^{2}\right)^{2} \text {. }
$$

The product formula is indeed independent of mass and depends on $n, \ell$, and $\nu$.

For a dyonic Bolt, we set $r_{ \pm}=r_{b}$ and satisfy the fourthorder equation for $r_{b}$ :

$$
6 n r_{b}^{4}-\ell^{2} r_{b}^{3}+2 n\left(\ell^{2}-3 n^{2}+\ell^{2} v^{2}\right) r_{b}^{2}-2 \ell^{2} v^{2} n^{3}=0
$$

Let $r_{b}=r_{b \pm}$ be the solution of the equation. Then we find the action as before:

$$
\begin{aligned}
I_{ \pm} & =-2 \pi \\
& \cdot \frac{\left[r_{b}^{4}-\ell^{2} r_{b}^{2}+n^{2}\left(3 n^{2}-\ell^{2}\right)\right] r_{b}^{2}-\left(r_{b}^{4}+4 n^{2} r_{b}^{2}-n^{4}\right) \ell^{2} v^{2}}{\left(3 r_{b}^{2}-3 n^{2}+\ell^{2}\right) r_{b}^{2}+\left(r_{b}^{2}-n^{2}\right) \ell^{2} v^{2}} .
\end{aligned}
$$

Similarly the entropy is given by

$$
S_{ \pm}=2 \pi \frac{\left[3 r_{b}^{4}+\left(\ell^{2}-12 n^{2}\right) r_{b}^{2}+n^{2}\left(\ell^{2}-3 n^{2}\right)\right] r_{b}^{2}+\left(r_{b}^{4}+4 n^{2} r_{b}^{2}-n^{4}\right) \ell^{2} v^{2}}{\left(3 r_{b}^{2}-3 n^{2}+\ell^{2}\right) r_{b}^{2}+\left(r_{b}^{2}-n^{2}\right) \ell^{2} v^{2}}
$$

After substituting the value of $r_{b \pm}$ in the entropy product formula, it seems that the product is independent of mass and depends on $n, \ell, v$ for dyonic Taub-Bolt instanton.

\section{Conclusion}

We have studied the mass-independent feature for various gravitational instantons. This universal feature gives us strong indication towards understanding the microscopic properties of $\mathrm{BH}$ entropy. It would be interesting if one considered the entropy product formula for other instantons like multiTaub-NUT, non-self-dual Taub-NUT, $S^{4}, C P^{2}, S^{2} \times S^{2}$, and twisted $S^{2} \times S^{2}$. We expect these instantons also to give us universal features.

\section{Conflicts of Interest}

The author declares that they have no conflicts of interest.

\section{References}

[1] J. D. Bekenstein, "Black holes and the second law," Lettere Al Nuovo Cimento Series 2, vol. 4, no. 15, pp. 737-740, 1972.

[2] J. D. Bekenstein, "Black holes and entropy," Physical Review. D. Particles and Fields. Third Series, vol. 7, pp. 2333-2346, 1973.

[3] J. D. Bekenstein, "Generalized second law of thermodynamics in black-hole physics," Physical Review D, vol. 9, no. 12, pp. 32923300, 1974.

[4] B. Carter, "Rigidity of a black hole," Nature Physical Science, vol. 238 , no. 83 , pp. 71-72, 1972.
[5] J. M. Bardeen, B. Carter, and S. W. Hawking, "The four laws of black hole mechanics," Communications in Mathematical Physics, vol. 31, pp. 161-170, 1973.

[6] S. W. Hawking, "Gravitational radiation from colliding black holes," Physical Review Letters, vol. 26, no. 21, pp. 1344-1346, 1971.

[7] S. W. Hawking, "Black hole explosions?" Nature, vol. 248, no. 5443, pp. 30-31, 1974.

[8] S. W. Hawking, "Particle creation by black holes," Communications in Mathematical Physics, vol. 43, no. 3, pp. 199-220, 1975.

[9] A. Strominger and C. Vafa, "Microscopic origin of the Bekenstein-Hawking entropy," Physics Letters. B. Particle Physics, Nuclear Physics and Cosmology, vol. 379, no. 1-4, pp. 99-104, 1996.

[10] F. Larsen, "String model of black hole microstates," Physical Review. D. Third Series, vol. 56, no. 2, pp. 1005-1008, 1997.

[11] M. Ansorg and J. Hennig, "Inner Cauchy horizon of axisymmetric and stationary black holes with surrounding matter in Einstein-Maxwell theory," Physical Review Letters, vol. 102, no. 22, Article ID 221102, 2009.

[12] M. Cvetic, G. W. Gibbons, and C. N. Pope, "Universal area product formulas for rotating and charged black holes in four and higher dimensions," Physical Review Letters, vol. 106, no. 12, Article ID 121301, 2011.

[13] A. Castro and M. J. Rodriguez, "Universal properties and the first law of black hole inner mechanics," Physical Review D, vol. 86, no. 2, Article ID 024008, 2012.

[14] M. Visser, "Area products for stationary black hole horizons," Physical Review D, vol. 88, no. 4, Article ID 044014, 2013.

[15] S. Detournay, "Inner mechanics of three-dimensional black holes," Physical Review Letters, vol. 109, no. 3, Article ID 031101, 2012. 
[16] B. Chen, S. Liu, and J.-J. Zhang, "Thermodynamics of black hole horizons and Kerr/CFT correspondence," Journal of High Energy Physics, vol. 2012, article 17, 2012.

[17] P. Pradhan, "Black hole interior mass formula," European Physical Journal C, vol. 74, no. 5, article 2887, 2014.

[18] P. Pradhan, "Area product and mass formula for Kerr-NewmanTaub-NUT spacetime," Modern Physics Letters A, vol. 30, no. 35, Article ID 1550170, 2015.

[19] P. Pradhan, "Thermodynamic product formula for HoravaLifshitz black hole," Physics Letters. B. Particle Physics, Nuclear Physics and Cosmology, vol. 747, pp. 64-67, 2015.

[20] P. Pradhan, "Entropy product formula for a spinning BTZ black hole," JETP Letters, vol. 102, no. 7, article 427, 2015.

[21] P. Pradhan, "Area (or entropy) product formula for a regular black hole," General Relativity and Gravitation, vol. 48, no. 2, article 19, 2016.

[22] P. Pradhan, "Area functional relation for 5D-Gauss-Bonnet-AdS black hole," General Relativity and Gravitation, vol. 48, no. 8, article 116, 2016.

[23] P. Pradhan, "Thermodynamic product relations for generalized regular black hole," Advances in High Energy Physics, vol. 2016, Article ID 8086740, 2016.

[24] P. Pradhan, "Thermodynamic products in extended phasespace," International Journal of Modern Physics. D. Gravitation, Astrophysics, Cosmology, vol. 26, no. 2, Article ID 1750010, 2017.

[25] P. Pradhan, "Logarithmic corrections in black hole entropy product formula," General Relativity and Gravitation, vol. 48, no. 7, article 98, 2016.

[26] P. Pradhan, "Thermodynamic products for Sen black hole," European Physical Journal C, vol. 76, no. 3, article 131, 2016.

[27] P. Pradhan, "Thermodynamic product formula for a Taub-NUT black hole," Journal of Experimental and Theoretical Physics, vol. 122, no. 1, pp. 113-117, 2016.

[28] P. Pradhan, "Surface area products for Kerr-Taub-NUT spacetime," EPL (Europhysics Letters), vol. 115, no. 3, Article ID 30003 , 2016.

[29] S. W. Hawking, "Gravitational instantons," Physics Letters. A, vol. 60 , no. 2 , pp. $81-83,1977$.

[30] G. W. Gibbons and S. W. Hawking, "Classification of gravitational instanton symmetries," Communications in Mathematical Physics, vol. 66, no. 3, pp. 291-310, 1979.

[31] T. Eguchi and A. J. Hanson, "Asymptotically flat self-dual solutions to euclidean gravity," Physics Letters B, vol. 74, no. 3, pp. 249-251, 1978.

[32] G. W. Gibbons and S. W. Hawking, "Action integrals and partition functions in quantum gravity," Physical Review D, vol. 15, no. 10, pp. 2752-2756, 1977.

[33] G. W. Gibbons and M. J. Perry, "New gravitational instantons and their interactions," Physical Review D, vol. 22, no. 2, pp. 313$321,1980$.

[34] D. N. Page, “Taub-NUT instanton with an horizon," Physics Letters B, vol. 78, no. 2-3, pp. 249-251, 1978.

[35] C. W. Misner, "The flatter regions of Newman, Unti, and Tamburino's generalized Schwarzschild space," Journal of Mathematical Physics, vol. 4, pp. 924-937, 1963.

[36] A. N. Aliev, H. Cebeci, and T. Dereli, "Kerr-Taub-NUT spacetime with Maxwell and dilaton fields," Physical Review D, vol. 77, no. 12, Article ID 124022, 2008.

[37] R. H. Boyer, "Geodesic killing orbits and bifurcate killing horizons," Proceedings of the Royal Society of London A: Mathematical, Physical and Engineering Sciences, vol. 311, pp. 245-252, 1969.
[38] S. W. Hawking and C. J. Hunter, "Gravitational entropy and global structure," Physical Review. D. Third Series, vol. 59, no. 4, Article ID 044025, 1999.

[39] D. N. Page and C. N. Pope, "Einstein metrics on quaternionic line bundles," Classical and Quantum Gravity, vol. 3, no. 2, pp. 249-259, 1986.

[40] D. N. Page and C. N. Pope, "Inhomogeneous Einstein metrics on complex line bundles," Classical and Quantum Gravity, vol. 4, no. 2, pp. 213-225, 1987.

[41] A. Chamblin, R. Emparan, C. V. Johnson, and R. C. Myers, "Large $N$ phases, gravitational instantons, and the nuts and bolts of AdS holography," Physical Review. D. Third Series, vol. 59, no. 6, Article ID 064010, 1999.

[42] R. B. Mann, "Misner string entropy," Physical Review. D. Third Series, vol. 60, no. 10, Article ID 104047, 1999.

[43] R. Emparan, C. V. Johnson, and R. Myers, "Surface terms as counterterms in the AdS-CFT correspondence," Physical Review. D. Third Series, vol. 60, no. 10, Article ID 104001, 1999.

[44] C. V. Johnson, "The extended thermodynamic phase structure of Taub-NUT and Taub-Bolt," Classical and Quantum Gravity, vol. 31, no. 22, Article ID 225005, 2014.

[45] J. F. Plebanski, "A class of solutions of Einstein-Maxwell equations," Annals of Physics, vol. 90, pp. 196-255, 1975.

[46] J. F. Plebanski and M. Demianski, "Rotating, charged, and uniformly accelerating mass in general relativity," Annals of Physics, vol. 98, no. 1, pp. 98-127, 1976.

[47] N. Alonso-Alberca, P. Meessen, and T. Ortin, "Supersymmetry of topological Kerr-Newman-Taub-NUT-AdS spacetimes," Classical and Quantum Gravity, vol. 17, no. 14, pp. 2783-2797, 2000.

[48] M. H. Dehghani and A. Khodam-Mohammadi, "Thermodynamics of Taub-NUT/bolt black holes in Einstein-Maxwell gravity," Physical Review. D. Third Series, vol. 73, no. 12, Article ID 124039, 2006 

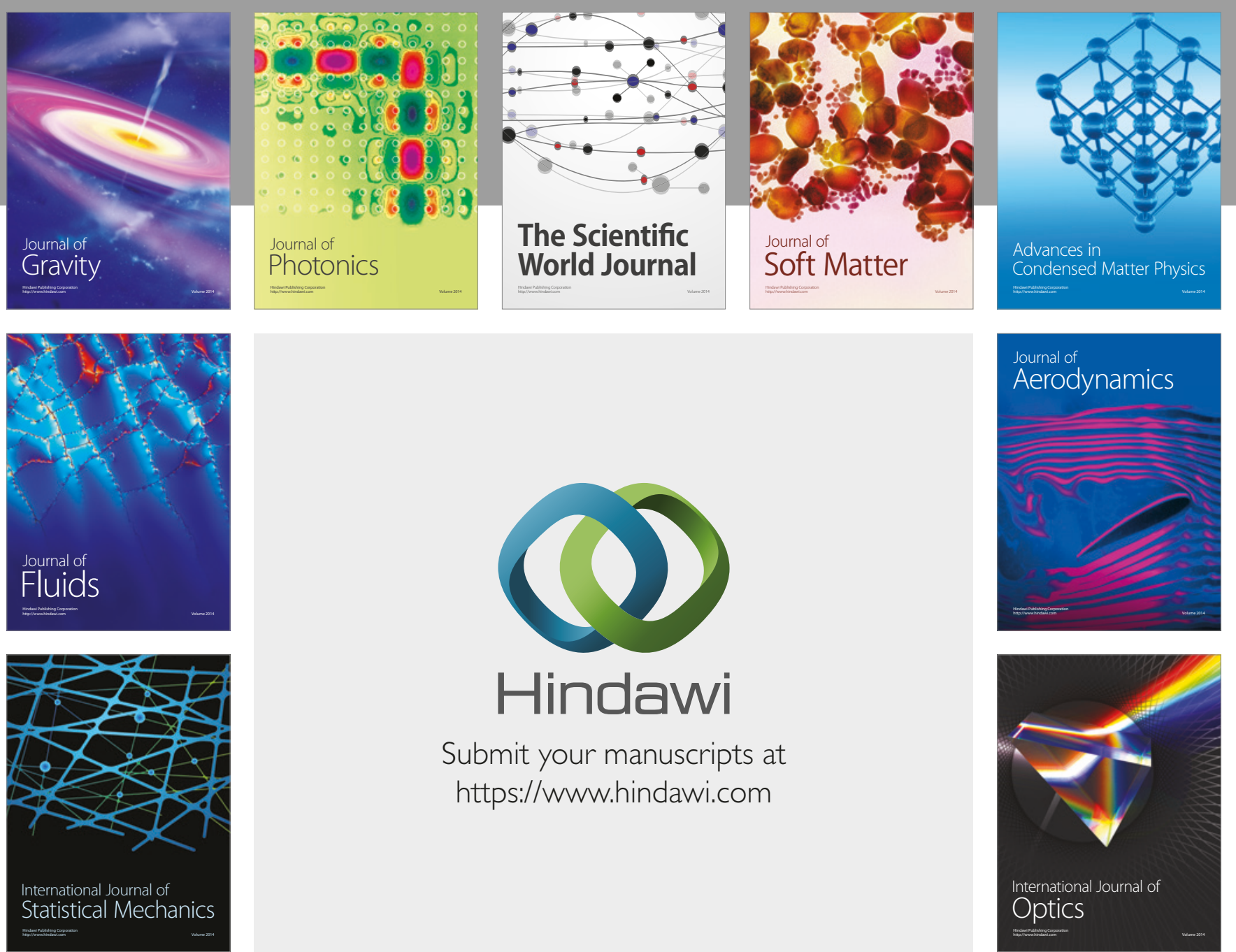

Submit your manuscripts at

https://www.hindawi.com
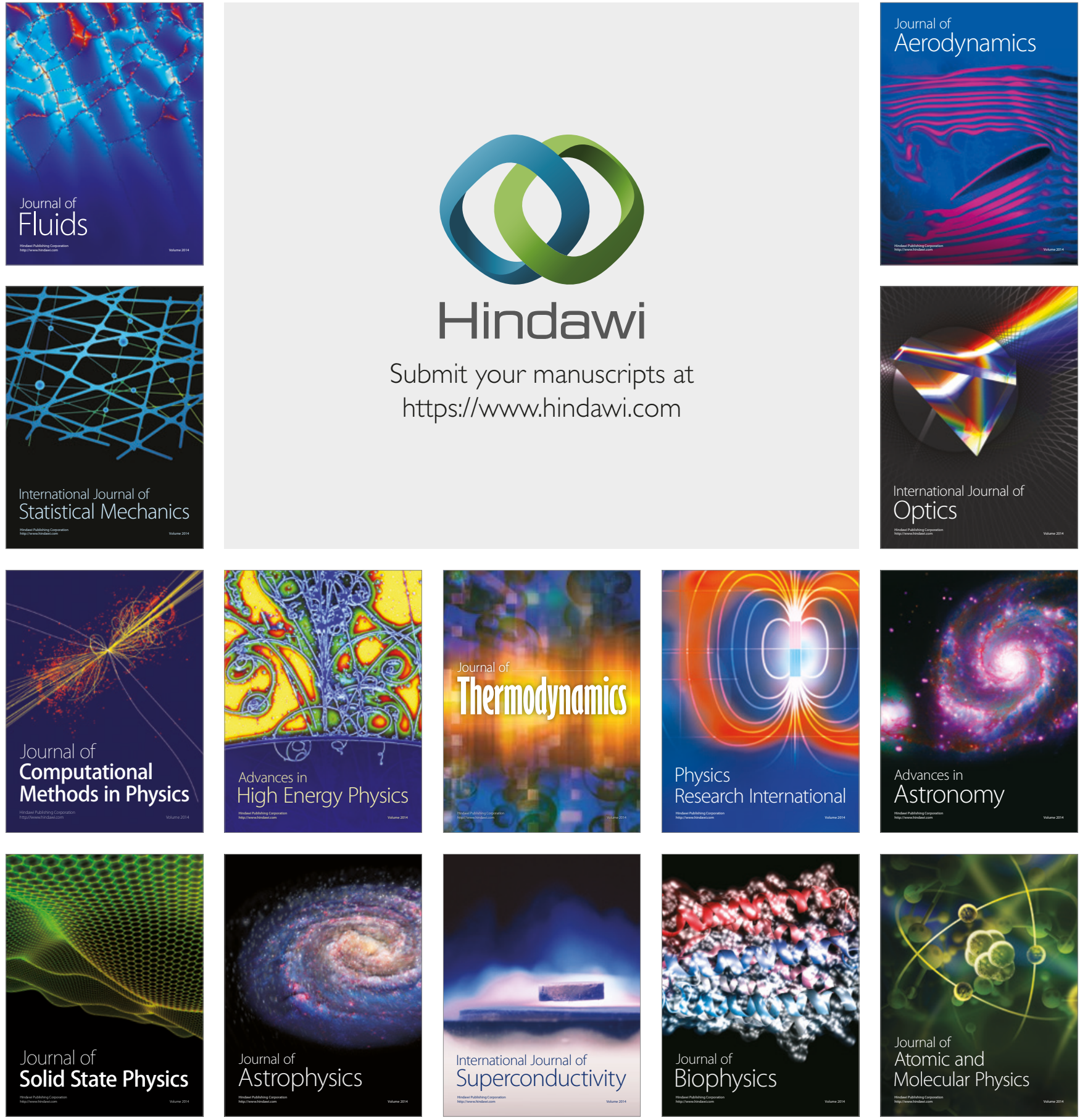